\title{
Dashboards for Progression Analysis of Students
}

\author{
Prabhleen Juneja ${ }^{1}$, Dr. Amardeep Singh ${ }^{2,}$ Dr. Kawaljeet Singh ${ }^{3}$ \\ ${ }^{1}$ M. Tech. Student, Department of Computer Engineering, Punjabi University, Patiala, India \\ ${ }^{2}$ Professor, Department of Computer Engineering, Punjabi University, Patiala, India \\ ${ }^{3}$ Director, University Computer Center, Punjabi University, Patiala, India
}

\begin{abstract}
The dashboard is a visualization device which shows the present status of metrics and Key Performance Indicators (KPIs) for an endeavor. They are a result of Business Intelligence i.e Descriptive Analytics. This paper aims to give an overview of what Dashboards are, what are their classifications and to introduce some common tools used to build the dashboards. The study also shows the parameters identified for measuring the student progression and performance of various departments of Engineering by describing student progression and data dimensional model. The purpose of a dashboard is to allow people to rapidly monitor relevant and critical information at a glance, render sufficient detail for context and analysis, and provide a fast and easy way of drilling-down to more facts. The dashboard is tailored for a specific role and display metrics are targeted for a single point of view of a department. It is a customizable interface and pulls real-time data from various sources.
\end{abstract}

Keywords: dashboard, dashboard types, big data, analytics, KPIs, Student Progression, Parameters, Data Dimensions, Multidimensional data model

\section{INTRODUCTION}

The dashboard is a visualization device which shows the present status of metrics and Key Performance Indicators (KPIs) for an endeavor. It shows the most imperative data expected to accomplish one or more destinations which fits completely on a solitary PC screen so it can be observed initially. Dashboards are relied upon to enhance choice amplifying so as to make cognizance and gaining by human perceptual abilities. Dashboard brings key metrics into a solitary showcase. Dashboards are intended to present information and causal connections that have as of now been recognized so they can be checked without requiring exertion or intuitiveness. In a solitary, dashboards just contain the most key information required to accomplish hierarchical destinations and tackle the issues that its expected clients face. The motivation behind a dashboard is to permit individuals to quickly screen important and basic data initially, give adequate subtle element to connection and investigation, and give a quick and simple method for boring down to more detail. Dashboard is a Knowledge change tool. Dashboards are helpful for the Visual presentation of execution measures, to perceive right and negative examples, to quantify efficiencies/inefficiencies, to make point by point reports showing new examples, to adjust strategies and legitimate targets, to spare time appeared differently in relation to running diverse reports and to pick up detectable quality of all structures in a split of a second.

1.1 Classification of Dashboards

Dashboards can be separated down according to role and dashboards are either strategic, or analytical, or operational, or informational.

- Strategic Dashboards

- Analytical Dashboards

- Operational Dashboards

- Performance Dashboards

1.2 Dashboard Architectures and Tools Used for the Development of Dashboards

The dashboard design comprises of the segments that involve the execution dashboard. The segments in every layer represent a superset of usefulness.

Different sorts of performance dashboards utilize diverse models. Operational dashboards tend to question source frameworks specifically and apply negligible changes, while strategic dashboards inquiry both historical and current data from an information stockroom. Strategic dashboards frequently make a neighborhood information store to reserve time series information for particular measurements. This segment gives an abnormal state 
examination of different information structures that support performance dashboards, including advantages and disadvantages.

Normally Direct Query, BI, Mashboards, In-Memory Dashboards, Data Federation, Data Marts and Complex Event Processing tools are used.

\subsection{Dashboard Performance Indicators}

Key Performance Indicator (KPI) is a type of performance measurement. An organization may use KPIs to evaluate its success, or to evaluate the success of a particular activity in which it is engaged. Sometimes success is defined in terms of making progress toward strategic goals, but often success is simply the repeated, periodic achievement of some levels of operational goal (e.g. zero defects, 10/10 customer satisfaction, etc. [1].

Key performance indicators, in practical terms and for strategic development, are objectives to be targeted that will add the most value to the business. These are also referred to as 'key success indicators'.

The Big Data Analytics concentrate on imaginative uses of handling huge information. It has moved past straightforward information mining to consider profound area learning that can be utilized by chiefs to deal with their associations all the more viably. Huge Data Analytics tackles the down to earth issues and exhibits the utility of Big Data in offering associations some assistance with delivering better data to their clients to enhance their choice making procedures.

\section{$1.4 \quad$ Student Progression and its Need}

Education today may be classified in three domains i.e cognitive domain, skill domain \& effective domain. Out of these cognitive domain is completely knowledge based and the behavioral patterns for students which are under this domain are termed as:

- Academic Behavior

- Social Behavior

- Leadership Behavior

- Application Behavior

Study of these behavioral patterns and detection of student's continuous advancement \& progress may be termed as Student Progression. It is also the student learning behavior depicting its simultaneous growth and development. Progression has indeed become an enormous factor of growth and success rate for various departments. Its essentiality depends upon the need of detecting the overall performance of students in a certain department which in turn helps improve the students' efficiency and finally their academic performance. With the help of study student progression and performance would be measured by specifying certain parameters. All these parameters have been kept in mind by maintaining the Data Quality as they exhibit real characteristics of data.

This paper aims to give an overview of what Dashboards are, what are their classifications and to introduce some common tools used to build the dashboards. The study also shows the student progression and performance analysis of various departments, on different parameters by describing the key performance indicators for measuring student progression and data dimensioning using OLAP cubes. On the basis of these parameters a Dashboard can be built.

\section{DIMENSIONAL DATA MODEL}

\subsection{Online Analytical Processing}

OLAP is the innovation behind numerous Business Intelligence (BI) applications. OLAP is an intense innovation for information revelation, including abilities for boundless report seeing, complex logical counts, and predict "what if" situation arranging. OLAP is PC handling that empowers a client to effectively and specifically concentrate and view information from various perspectives. To encourage this sort of analysis, OLAP information is put away in a multidimensional database. While a relational database can be considered as two-dimensional, a multidimensional database considers every information property, (for example, item, geographic deals district, and time period) as a different "dimension." OLAP programming can find the crossing point of dimensions and showcase them. 


\section{$2.2 \quad$ OLAP Cube}

An OLAP cube is a multidimensional database that is enhanced for data warehouse and online explanatory handling (OLAP) applications. An OLAP cube is a strategy for putting away information in a multidimensional structure, for the most part to report purposes. In OLAP cubes, information (measures) are ordered by dimensions. OLAP cubes are regularly pre-compressed crosswise over measurements to definitely enhance question time over social databases. The query language used to associate and perform assignments with OLAP cubes is multidimensional expressions (MDX).

\subsection{Data Dimensioning}

Dimensions are an essential piece of OLAP. Since OLAP is on-line, it must give answers rapidly; examiners posture iterative questions amid intelligent sessions, not in bunch employments that run overnight. What's more, in light of the fact that OLAP is likewise investigative, the questions are perplexing. The dimensional items and the OLAP motor are intended to fathom complex questions progressively.

The dimensional articles incorporate 3D squares, measures, measurements, traits, levels, and progressions. The effortlessness of the model is natural since it characterizes objects that speak to true business substances. Experts know which business measures they are keen on looking at, which measurements and qualities make the information significant, and how the measurements of their business are sorted out into levels and orders.

\section{$2.4 \quad$ Overview of the Dimensional Data Model}

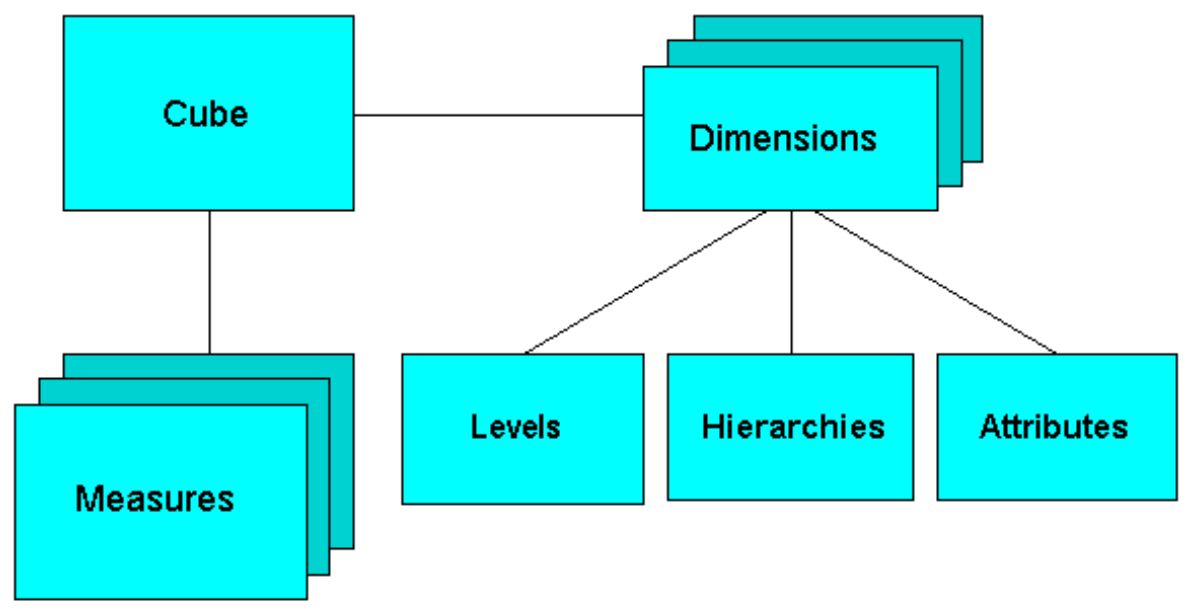

Fig. 1: OLAP Dimensional Model Diagram

The dimensional data model is exceptionally organized. Structure infers decides that oversee the connections among the information and control how the information can be questioned. cubes are the physical usage of the dimensional model, and hence are profoundly advanced for dimensional questions. The OLAP engine influences this natural dimensionality in performing very productive cross-cube joins for between column estimations, external joins for time arrangement investigation, and indexing. Measurements are pre-joined to the measures. The innovation that underlies blocks depends on a filed multidimensional array model, which gives direct cell access.

The OLAP engine controls dimensional articles similarly that the SQL engine controls social items. In any case, in light of the fact that the OLAP motor is streamlined to compute investigative capacities, and dimensional articles are enhanced for examination, logical and row functions can be ascertained much quicker in OLAP than in SQL.

The dimensional model empowers Oracle OLAP to bolster top of the line business insight apparatuses and applications, for example, OracleBI Discoverer Plus OLAP,OracleBI Spreadsheet Add-In, OracleBI Suite Enterprise Edition, BusinessObjects Enterprise, and Cognos ReportNet. 


\section{$2.5 \quad$ Cubes}

Cubes give a method for sorting out measures that have the same shape, that is, they have precisely the same. Measures in the same cube can without much of a stretch be broken down and showed together. A cube more often than not compares to a solitary truth table or view.

\subsection{Star Schemas \& OLAP Cubes}

Star schemas are dimensional structures sent in a social database administration framework (RDBMS). They naturally comprise of certainty tables connected to related measurement tables by means of essential/outside key connections. An online analytical processing (OLAP) cube is a dimensional structure executed in a multidimensional database; it can be proportional in substance to, or all the more frequently got from, a relational star schema. An OLAP cube contains dimensional attributes and facts, yet it is gotten to through dialects with more scientific abilities than SQL, for example, XMLA. OLAP cubes are incorporated into this rundown of essential methods in light of the fact that an OLAP cube is regularly the last step in the sending of a dimensional DW/BI framework, or might exist as a total structure taking into account a more nuclear relational star schema [14].

\subsection{Multidimensional Data Model}

By incorporating multidimensional articles and examination into the database, the force of multidimensional investigation alongside the dependability, accessibility, security, and adaptability can be accomplished.

- Dimensional items can be are put away in Database in their local multidimensional arrangement.

- Cubes and other dimensional items are five star information objects spoke to in the information word reference.

- Data security is controlled in the standard path, by giving and denying benefits to Database clients and parts.

- Applications can inquiry dimensional items utilizing SQL.

The advantages of this are critical. Database OLAP offers the force of straightforwardness: One database, standard organization and security, standard interfaces and advancement devices. Analysts can pick any SQL inquiry and examination device for selecting, seeing, and breaking down the information. You can utilize your most loved tool or application, or utilize an instrument supplied with Oracle Database.

This dashboard shows data rich counts, for example, proportion, offer, former period, and total aggregate. Separate tabs on the dashboard present Profitability Analysis, Sales Analysis, and Product Analysis. Every tab introduces the information in dials, bar graphs, flat bar outlines, pie diagrams, and cross-plain reports. A drop-down rundown in the upper left corner gives a decision of Customers.

The dial shows the quarterly net revenue. To the privilege is a bar diagram that contrasts current benefits and year-ago benefits.

\section{PARAMETERS IDENTIFIED}

3.1 In order to design the Performance Dashboard for the Department of Computer Engineering, Punjabi University, Patiala, following parameters have been identified for measuring the progression \& performance of Students.

- Student Academic Performance(Attendance)

- Monitoring of Student performance in MST/Final Semester Exams

- Average time spent by students(weekly)

- Discipline

- Students who attend workshop/seminar

- Lab-Performance

- $\quad$ Result (Last 3 years)

- Internet/wi-fi facilities in lab/hostel

- Geographical distribution of Students 
3.2 Multidimensional Data Model

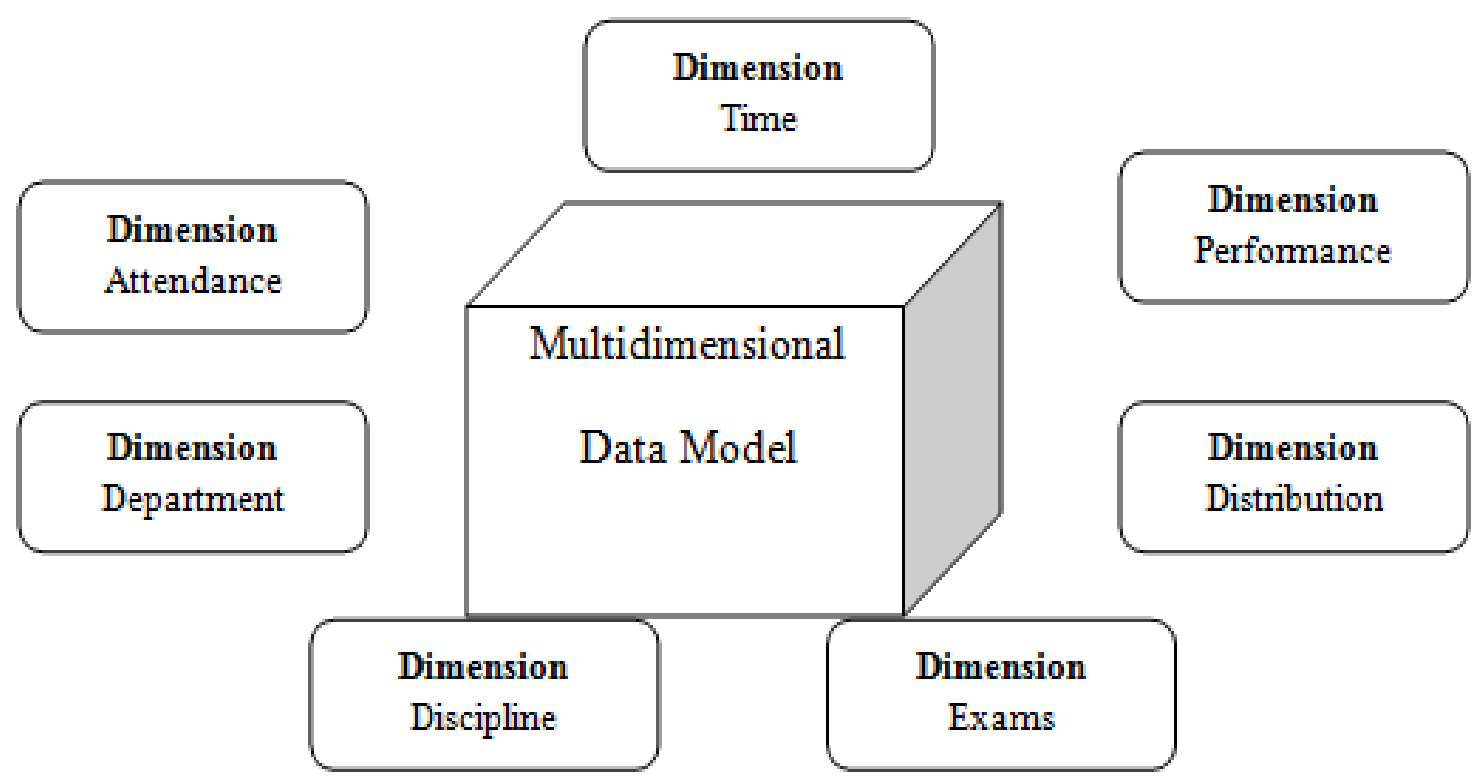

Fig. 2: A Multidimensional Data Model for Measuring Progression \& Performance of Students

Dashboards can be designed using Multidimensional Technology with the latest DBMS packages like Oracle.

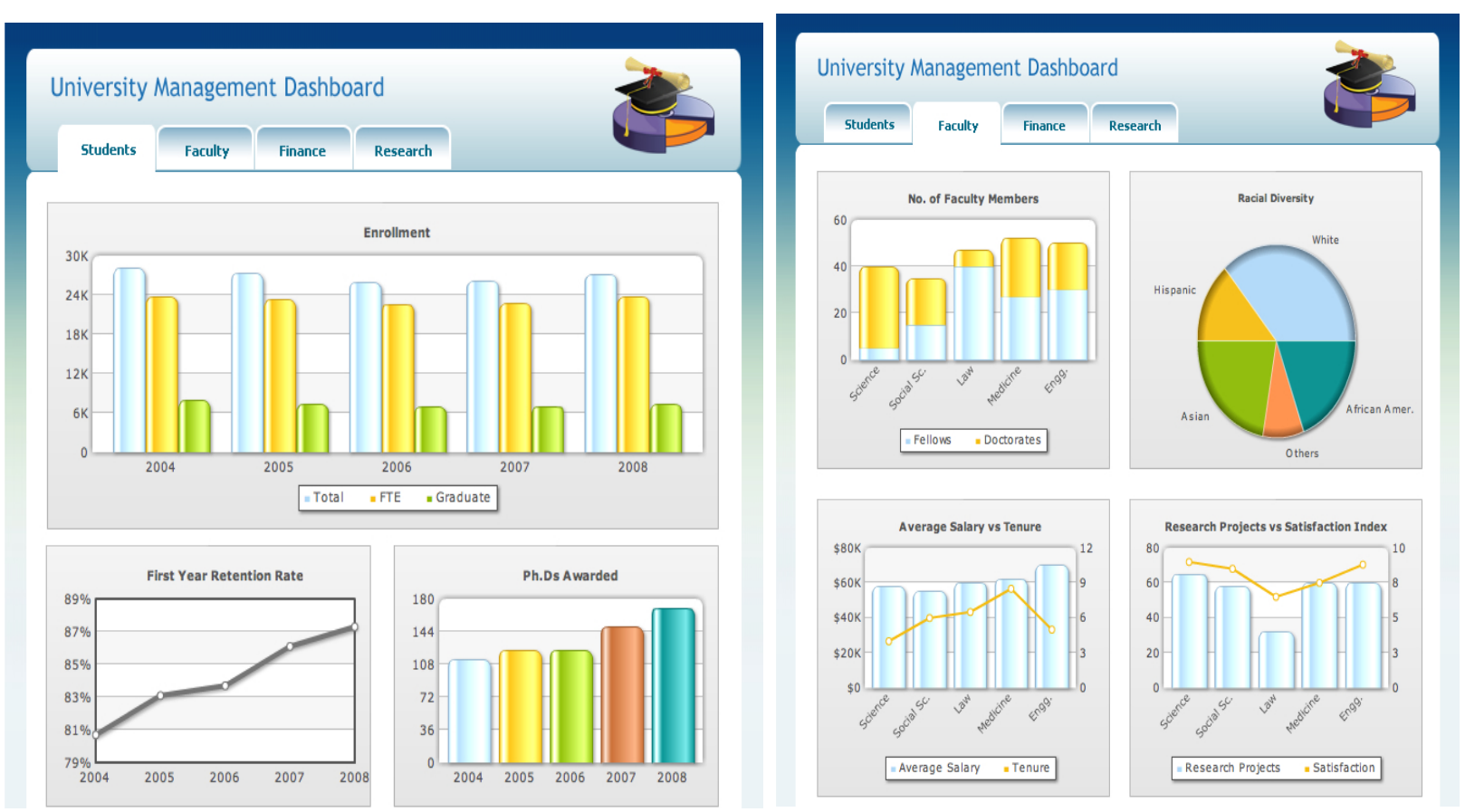




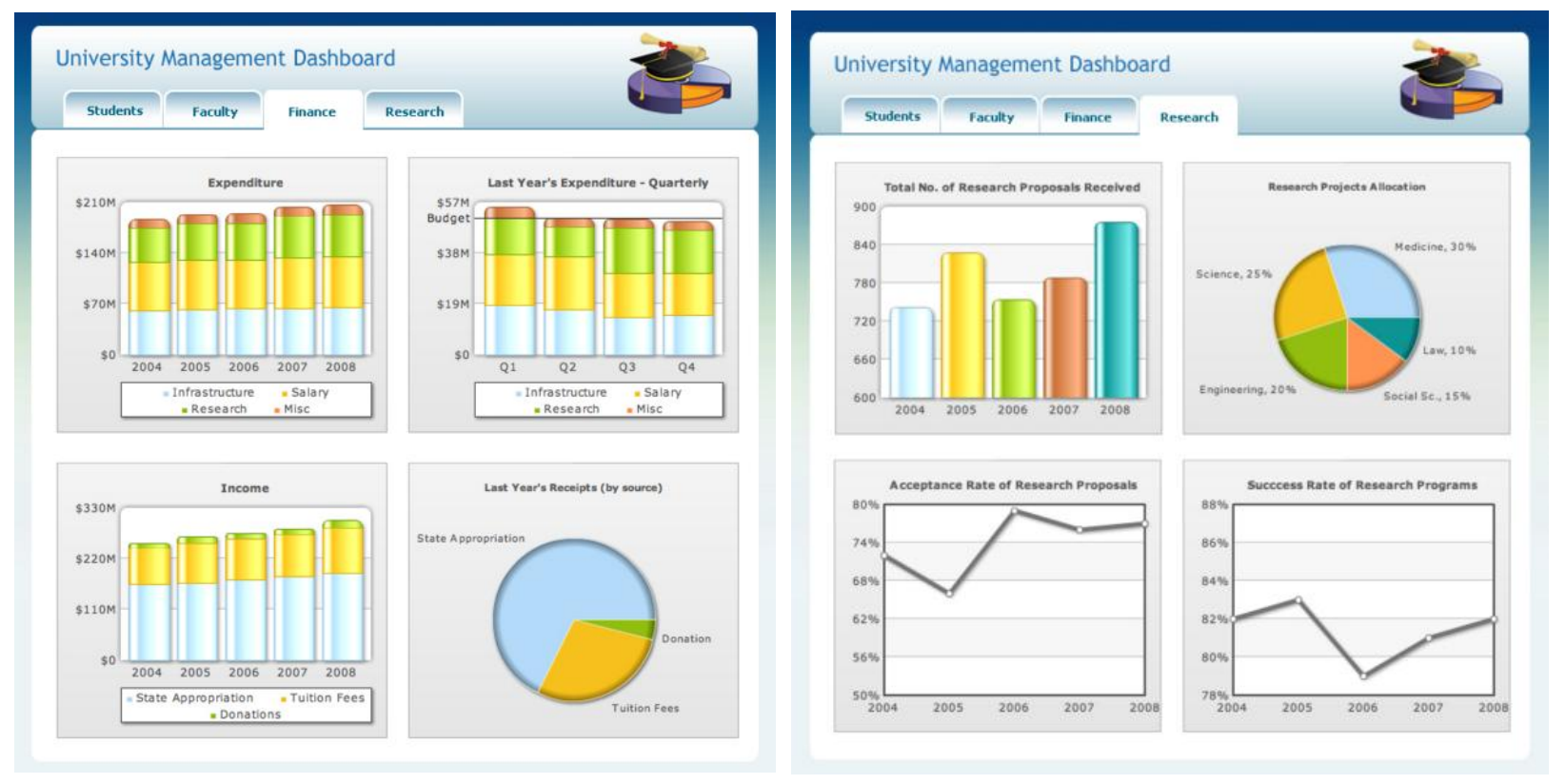

Fig. 3: Sample Dashboards for Universities

\section{CONCLUSION}

Various organizations deal with the problem of a limited amount of information to impart to their employees which further results in delayed decision making, low efficiency, lesser output, higher costs for production and manufacturing and finally in poor customer service.

This paper presents a detailed introduction on Dashboards with an emphasis on the types of Dashboards, their architecture and tools. It is also described that by dimensioning the data into various schemas and OLAP cubes can create a multidimensional model according to our need. A diagrammatic view of multidimensional model of our survey has also been depicted along with the parameters identified for creating a dashboard. By studying the features of Dashboards it is concluded that for any organization it is very crucial to determine the success and failure of the company. The dashboard designed should be in real-time, user-friendly, include background and future data, which should be depicted by graphs, charts, tabular tables, gauges, indicators for a better display and visual. The Dashboard should have an interface for knowledge, supplier and customer. This helps the decision maker make wiser and faster business decisions

Thus, the features and types discussed above would help us to develop a framework for dashboards which are to be used in the creation of dashboards for specific departments of Engineering. Depending upon the requirements of the organizations, the features can be added in, in order to improve the efficiency and performance of students. The major challenge in the field of Dashboards is that how to choose appropriate parameters in order to depict an organizations strengths and weaknesses. So, various experimental scenarios are needed to be considered for the same.

\section{REFERENCES}

[1] R.N.Prasad and Seema Acharya, Basics of Enterprise Reporting, Fundamentals of Business Analytics, Reprint Edition. New Delhi, Wiley, 2014

[2] Ephraim Turban, Ramesh Sharda, Dursun Delen and David King , Business Intelligence: A Managerial Approach, 2 ${ }^{\text {nd }}$ Edition,Pearson

[3] Mohamed Addelfattah, A Comparison of Several Performance Dashboards Architectures, Intelligent Information Management, vol. 5,pp. 35-41, March 2013

[4] Ernie Mazuin Mohd Yusof and Mohd Shahizan Othman, A review on the Dashboard Characteristics for Manufacturing Organizations, Journal of Information Systems Research and Innovation, ISSN: 2289-1358, 2012 
[5] Koen Pauwels, Tim Ambler, Bruce H.Clark, Pat LaPointe, David Reibstein, Bernd Skiera, Berend Wierenga and Thorsten Wiesel, Dashboards as a Service: Why, What, How and What research Is Needed?, Journal of Service Research, vol. 12 (2), pp.175-189, November 2009

[6] Mihaela Muntean, Gheorghe Sabau, Ana-Ramona Bologa, Traian Surcel and Alexandra Florea, Performance Dashboards for Universities, Proc $2^{\text {nd }}$ International Conference on Manufacturing Engineering, Quality and Production Systems, ISSN: 1792-4693, ISBN: 978960-474-220-2, 2010

[7] Katrien Verbert, Stan Govaerts, Erik Duval, Jose Luis Santos, Frans Van Assche, Gonzalo Parra and Joris Klerkx, Learning dashboards: an overview and future research opportunities, Pers Ubiquit Comput(2014), Springer-Verlag London, vol. 18, pp. 1499-151

[8] Meyliana, Henry A. E Widjaja, Stephen W. Santoso, University dashboard: An implementation of executive dashboard to university, Information and Communication Technology (ICoICT), 2014 2nd International Conference, 978-1-4799-3580, pp., 282 - 287, 28-30 May 2014

[9] Nathalie Cassaigne, The Dashboard: a knowledge conversion tool, Engineering Management Conference, 2002. IEMC '02. 2002 IEEE International , 0-7803-7385, vol. 1 ,pp. $292-297,2002$

[10] Duong Thi Anh Hoang, Thanh Binh Nguyen, A Min Tjoa, Dashboard by-example: A hypergraph-based approach to on-demand data warehousing systems, Systems, Man, and Cybernetics (SMC), IEEE International Conference, 978-1-4673-1714, pp. 1853 - 1858, 14-17 Oct. 2012

[11] Ralf Gitzel, Simone Turrin, Sylvia Maczey, A Data Quality Dashboard for Reliability Data, Business Informatics (CBI), IEEE 17th Conference, 978-1-4673-7340, vol. 1, pp. 90 - 97, July 2015

[12] Mohammed M. Abu Tair, Alaa M. El-Halees, Mining Educational Data to Improve Students' Performance:A Case Study, International Journal of Information and Communication Technology Research, ISSN 2223-4985, Vol 2 No. 2, pp. 140 - 146, Feb 2012

[13] Jeff Reinking, The Diffusion of Digital Dashboards: An Examination of Dashboard utilization and the managerial decision environment, Degree of Doctor of Philosophy at the University of Central Florida, 2013

[14] Ralph Kimball and Margy Ross, Kimball Dimensional Modeling Techniques, Kimball Group, 2013

[15] Pratiyush Guleria and Manu Sood, Data Mining in Education: A review on the knowledge Discovery Perspective, International Journal of Data Mining \& Knowledge Management Process (IJDKP) Vol.4 (5), Sept 2014

[16] Darrell M. West, Big Data for Education: Data Mining, Data Analytics, and Web Dashboards , Governance Studies at Brookings, Sept 2012

[17] Katrien Verbert, Erik Duval, Joris Klerkx, Sten Govaerts, and José Luis Santos, Learning Analytics Dashboard Applications, American Behavioral Scientist, Sage Publications, pp. 1- 10, 2013

[18] Lindsey Wise, Big data - what it means for data visualization and dashboard applications, http://www.dashboardinsight.com/articles/new-concepts-in-business-intelligence/big-data-what-it-means-for-data-visualization-and-dashboardapplications.aspx, September 01, 2011

[19] Jeff Bertolucci, Big Data Analytics: Descriptive Vs. Predictive Vs. Prescriptive, http://www.informationweek.com/big-data/big-dataanalytics/big-data-analytics-descriptive-vs-predictive-vs-prescriptive/d/d-id/1113279,December 31,2013 